\title{
Echocardiographic Evaluation of the Thoracic Aorta: Tips and Pitfalls
}

\author{
Sarah C. Hull, MD, MBE ${ }^{10}$ \\ ${ }^{1}$ Section of Cardiovascular Medicine, Columbia St. Mary's Hospital, \\ Milwaukee, Wisconsin \\ 2 Division of Cardiovascular Diseases, University of Tennessee- \\ Methodist, Knoxville, Tennessee \\ ${ }^{3}$ Section of Cardiovascular Medicine, Yale University School of \\ Medicine, New Haven, Connecticut
}

Krishna Upadhyaya, $\mathrm{MD}^{1}$ If Ifeoma Ugonabo, MD, $\mathrm{MPH}^{2}$ Keyuree Satam, BA

Aorta (Stamford) 2021;9:1-8.

\begin{abstract}
Address for correspondence Krishna Upadhyaya, MD, Section of Cardiovascular Medicine, Columbia St. Mary’s Hospital, 2350 N Lake Dr, Suite 400, Milwaukee, WI 53211 (e-mail: krishnau26@gmail.com).
\end{abstract}

\begin{abstract}
Keywords

- echocardiography

- aorta

- dissection

- bicuspid aortic valve

By convention, the ascending aorta is measured by echo from leading edge to leading edge. "Leading edge" connotes the edge of the aortic wall that is closest to the probe (at the top of the inverted " $\mathrm{V}$ " of the ultrasound image). By transthoracic echo (TTE), the leading edges are the outer anterior wall and inner posterior wall. By transesophageal echo (TEE), the leading edges are the outer posterior wall and inner anterior wall. Aortic measurements should be taken (by convention) in diastole (when the aorta is moving least). Simple TTE is 70 to $85 \%$ sensitive in diagnosing ascending aortic dissection. TEE sensitivity approaches $100 \%$, though the tracheal carina imposes a blind spot on TEE, impeding visualization of distal ascending aorta and proximal aortic arch. While computed tomography angiography may be superior for defining full anatomic extent of aortic dissection, echocardiography is superior in assessing functional consequences such as mechanism and severity of aortic regurgitation, evidence of myocardial ischemia when complicated by coronary dissection, or evidence of tamponade physiology when pericardial effusion is present. Reverberation artifact can mimic a dissection flap. A true flap moves independently of the outer aortic wall which can be confirmed by M-mode. Color flow respects a true flap but does not respect a reverberation artifact. Assessment for bicuspid aortic valve (BAV) morphology should be done in systole, not diastole. In diastole, when the valve is closed, the raphé can make a bicuspid valve appear trileaflet. Doming in the parasternal long axis (PLAX) view and an eccentric closure line on PLAX M-mode should also raise suspicion for BAV.
\end{abstract}

\section{Introduction}

Aortic disease can present as a multitude of conditions, ranging from asymptomatic aneurysms to life-threatening acute aortic syndromes. While a significant cause of morbidity and mortality, the incidence of aortic disease is likely

received

July 3, 2020

accepted after revision

November 11, 2020
DOI https://doi.org/

$10.1055 / \mathrm{s}-0041-1724005$.

ISSN 2325-4637. underestimated due to some processes being clinically silent. Thoracic aortic aneurysms (TAA) are estimated to have an annual incidence of 5 to 10 cases $/ 100,000$ patient years, with more acute symptomatic syndromes like thoracic aortic dissection having an incidence of 3 to 4 cases/100,000
(C) 2021. The Author(s).

This is an open access article published by Thieme under the terms of the Creative Commons Attribution License, permitting unrestricted use, distribution, and reproduction so long as the original work is properly cited. (https://creativecommons.org/licenses/by/4.0/)

Thieme Medical Publishers, Inc., 333 Seventh Avenue, 18th Floor, New York, NY 10001, USA 
patient years. ${ }^{1,2}$ In acute aortic syndromes, and especially in asymptomatic diseases where there may not be any physical examination cues, echocardiography plays an important role in diagnosis and serial evaluation. Standard transthoracic echocardiography (TTE) obtains certain views of the aorta and is useful not only in diagnosis but also in follow-up screening. Transesophageal echo (TEE) also plays a vital role in obtaining a more comprehensive view of the aorta, especially when transthoracic views are limited. In this review, we will highlight echocardiographic evaluation of the normal aorta and selected aortic pathologies, including tips to improve imaging quality and pitfalls to avoid that may impede accurate assessment.

\section{Echocardiographic Evaluation of the Normal Aorta}

The aorta is divided into five anatomic segments: (1) aortic root, (2) tubular ascending aorta, (3) aortic arch, (4) descending aorta, and (5) abdominal aorta. The aortic root is further subdivided into the sinuses of Valsalva and the sinotubular junction, which is the intersection between the root and the tubular ascending aorta. The aortic annulus is the most proximal portion of the aortic root, where the leaflets of the aortic valve are inserted. Just distal to that are the sinuses of Valsalva from which the coronary arteries originate. The aorta as a whole serves as a dynamic elastic conduit connecting the cardiac pump to the rest of the organs in the body and thereby delivers oxygenated blood. Evaluation of the aorta is part of the standardized protocol when performing a TTE, though it is important to note that not every aforementioned segment may be adequately visualized with this modality.

The canonical views in which the aorta is visualized with TTE are the parasternal long axis (PLAX), parasternal short axis, apical view, subcostal view, and suprasternal view, typically in that order. ${ }^{3}$ The PLAX is viewed by placing the patient in the left lateral decubitus position and the ultrasound probe at the third or fourth intercostal space, adjacent to the sternum on the left side, with the transducer marker pointing to the right shoulder. In this window, the parts of the aorta visualized in longitudinal cross-section are the aortic annulus, sinuses of Valsalva, sinotubular junction, and proximal part of the tubular ascending aorta ( $\mathbf{- F i g . 1 A})$. The descending aorta can also be visualized just below the left atrium, though in transverse cross-section. A tip to better visualize the tubular ascending aorta is remaining adjacent to the sternum but going up a rib space. This window allows for better visualization and is used for measurement of the

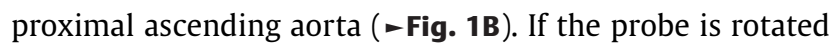
90 degrees with the marker facing the left shoulder, the left ventricle appears in transverse cross section (short axis), and if the probe tail is tipped down, the aortic annulus appears in transverse cross-section with visualization of the aortic valve (-Fig. 1C). This is useful in helping evaluate the number of aortic valve leaflets. In the apical five and three chamber view, the aortic annulus and sinuses of Valsalva can be visualized (-Fig. 1D), though size measurements are not
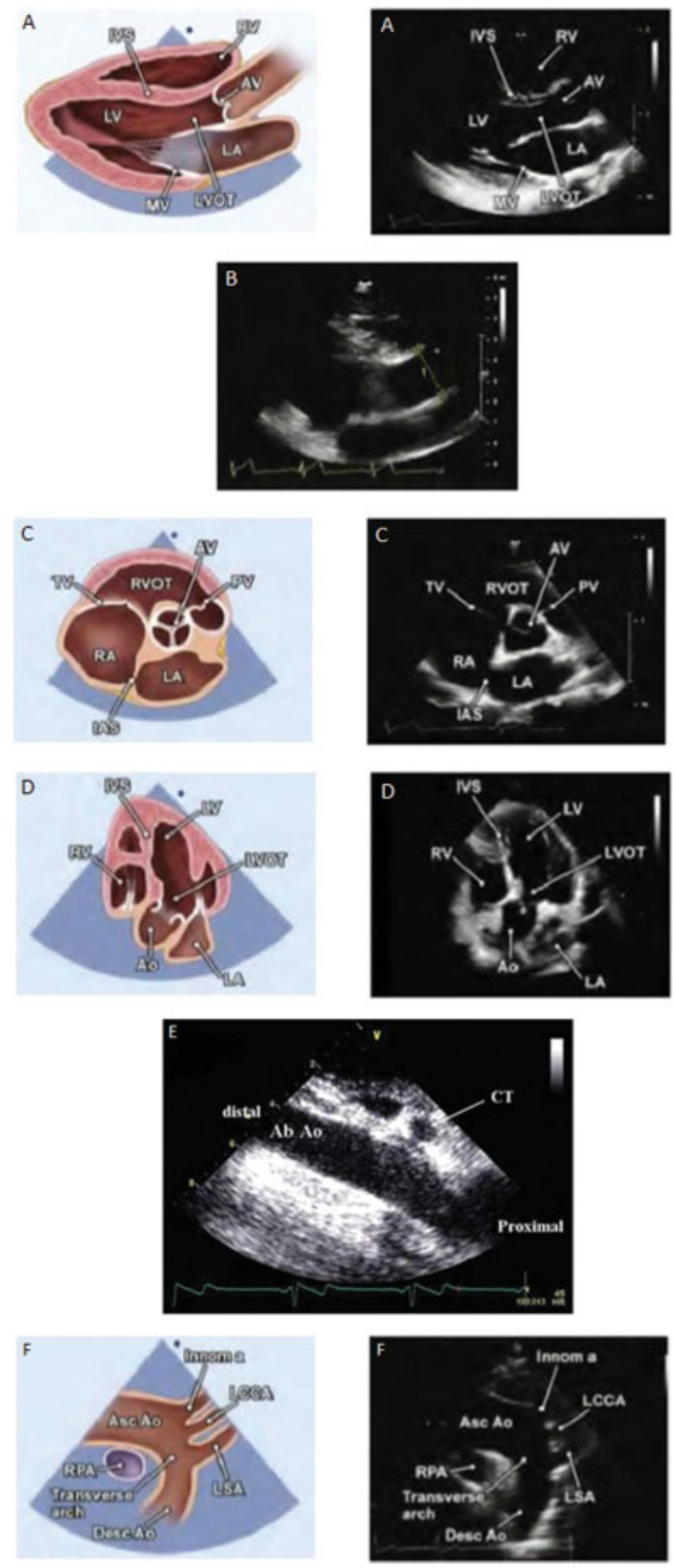

Fig. 1 Canonical views on transthoracic echo (TTE): (A) parasternal long axis with anatomic and TTE images, (B) parasternal long axis TTE image up a rib space showing ascending aorta, (C) parasternal short axis with anatomic and TTE images, (D) apical five chambers with anatomic and TTE images, (E) subcostal TTE image, (F) suprasternal with anatomic and TTE images. Images (A-D) and (F) adapted from Mitchell et al ${ }^{3}$ and image (E) from Evangelista et al. ${ }^{13} \mathrm{Ab}$ Ao, abdominal aorta; Asc Ao, ascending aorta; AV, aortic valve; $\mathrm{CT}$, computed tomography; Desc Ao, descending aorta; IAS, interartrial septum; Innom a, innominate artery; IVS, interventricular septom; LA, left atrium; LAX, long axis; LCCA, left common carotid artery; LSA, left subclavian artery; LV, left ventricle; LVOT, left ventricular outflow tract; ME, mid esophageal; MV, mitral valve; PV, pulmonic valve; RA, right atrium; RV, right ventricle; RVOT, right ventricular outflow tract; SAX, short axis; TV, tricuspid valve; UE, upper esophageal. 
typically made in this view as the PLAX is preferred given closer proximity to the probe. This window is useful in obtaining functional information about the valve, such as stenosis or regurgitation, given better alignment with the spectral Doppler interrogation. The subcostal view is helpful in evaluating the descending aorta, which can be visualized by tipping the probe tail up after seeing the inferior vena cava with the marker pointing in the 3 o'clock position (-Fig. 1E). Finally, in the suprasternal view, the aortic arch can be visualized, including the three main branches of the arch if image quality is excellent (-Fig. 1F).

The canonical views in which the aorta is visualized on TEE include mid-esophageal and upper-esophageal views at
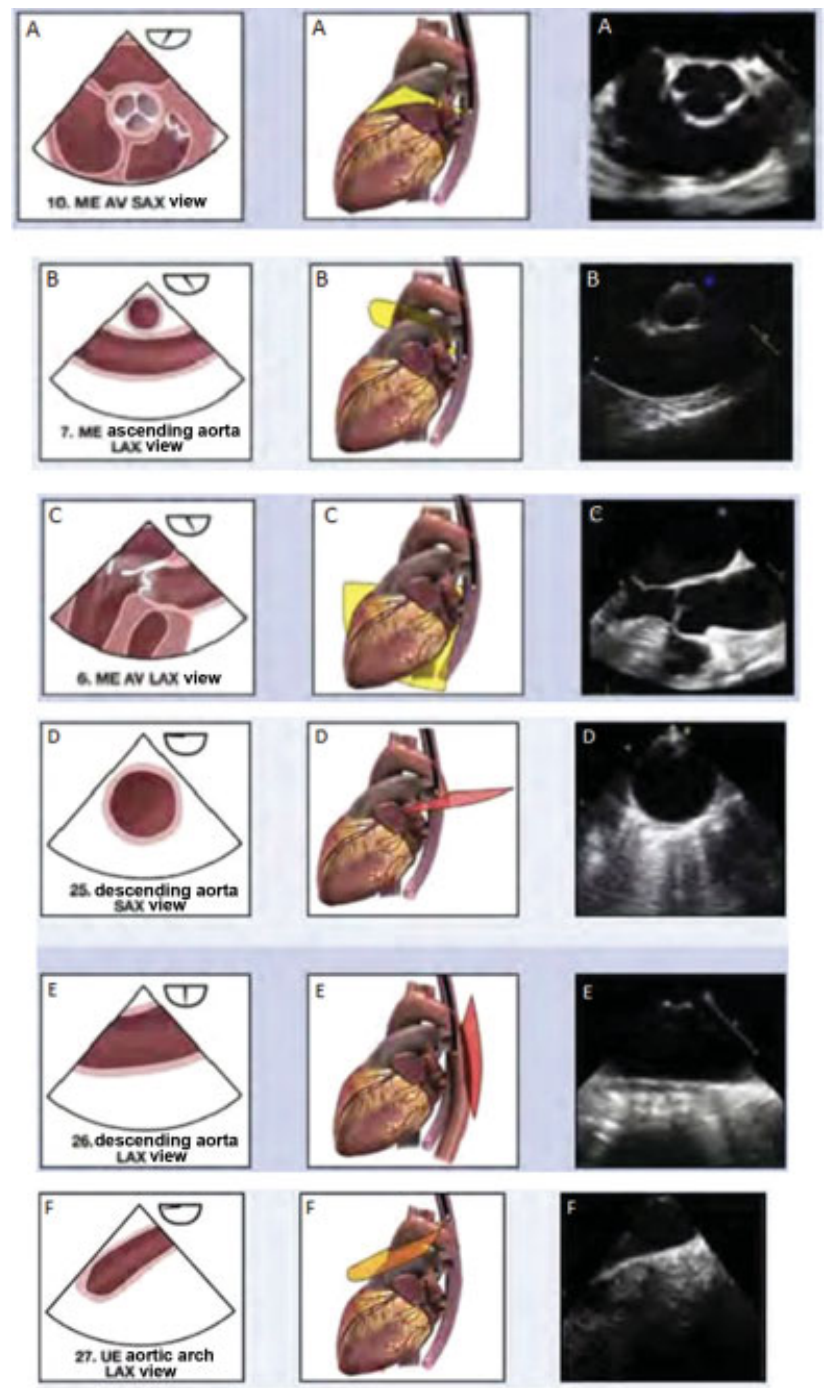

Fig. 2 Canonical views on transesophageal echo (TEE): (A) mid-esophageal view at 45 degrees (short axis) with anatomic, three-dimensional (3D) and TEE images, (B) mid-esophageal view at 100 degrees of the ascending aorta with anatomic, 3D and TEE images (C) mid-esophageal view at 135 degrees (long axis) with anatomic, 3D and TEE images, (D) mid-esophageal view at 0 degrees (short axis) of the descending aorta with anatomic, 3D and TEE images, (E) mid-esophageal view at 90 degrees (long axis) of the descending aorta with anatomic, 3D and TEE images, $(F)$ upper esophageal view at 0 degrees of the aortic arch with anatomic, 3D and TEE images. AV, aortic valve; LAX, long axis, ME, mid esophageal; SAX, short axis; UE, upper esophageal. Image courtesy: Hahn et al. ${ }^{4}$ different plane angles. ${ }^{4}$ At approximately 45 degrees in the mid-esophageal view, the aortic valve can be seen in transverse cross-section, which is helpful for direct visualization to determine the number of leaflets ( $\mathbf{- F i g . ~} \mathbf{2 A}$ ). If the probe is pulled out a few centimeters, at approximately 100 degrees and with some anteflexion, the proximal ascending aorta can be laid out in a longitudinal view for diameter measurement (-Fig. 2B). In the mid-esophageal view at 135 degrees, the aortic valve can be visualized in long axis, which can be used to measure the left ventricular outflow tract, sinuses of Valsalva, and sinotubular junction, as well as to assess aortic valve function (-Fig. 2C). Finally, in the mid-esophageal view, the probe is rotated in clockwise or counterclockwise fashion until the descending aorta can be viewed, in 0 degrees as short axis and 90 degrees as long axis (-Fig. 2D, E). As the probe is pulled out, in the upper esophagus, the aortic arch can be visualized as well (-Fig. 2F).

When measuring the aorta by echocardiography, it is important to emphasize that normal aortic size can vary from person to person based on certain patient characteristics. Roman et $\mathrm{al}^{5}$ studied the aortic root diameters of 135 normal adults and 52 normal infants and children and compared several characteristics including age, gender, body habitus, blood pressure, and stroke volume. Of these characteristics, age and body surface area (BSA) were found to be the most significant factors in determining normal aortic size. - Fig. 3 shows normal ranges of the sinuses of Valsalva diameter for three different age groups: - Fig. $\mathbf{3 A}$ is for ages 1 to 15 years, - Fig. 3B is for 20 to 39 years, and -Fig. $3 \mathrm{C}$ is for $\geq 40$ years. Based on this chart, for example, a 50-year-old patient with a BSA of 2.2 would have an upper limit of normal of $4.2 \mathrm{~cm}$, whereas a 30 year-old patient with a BSA of 1.6 would have an upper limit of normal of $3.2 \mathrm{~cm}$.

Normal values of the ascending aorta are also based on certain patient characteristics. Davies et $\mathrm{al}^{6}$ followed 803 patients with thoracic aortic aneurysms with serial multimodal imaging and found that aortic diameter indexed to BSA was a more important predictor of outcomes than absolute diameter. In a more recent study, the same group indexed aortic size to height in 780 patients and found that height-based ratio was as good or better at predicting outcomes (-Fig. 4). ${ }^{7}$ Therefore, it is important not to fall into the pitfall of "one size fits all" for normal aortic size dimensions.

It is also important to emphasize the proper technique for measuring the normal size of the aorta. There are three key techniques that should be followed to ensure accurate measurement: (1) timing of the cardiac cycle, (2) edges of measurement, and (3) echocardiographic windows. ${ }^{8}$ With respect to timing of the cardiac cycle, all measurements of the aorta except the aortic annulus are recommended to be done in end diastole. The aorta has elastic properties that enable it to accommodate the ejection of blood from the left ventricle, and thus varies slightly in diameter between systole and diastole. Therefore, consistent measures must be used to compare with normal values for each study, as well as to compare follow-up studies in a single patient. With respect to edges of measurements, aortic root and ascending 

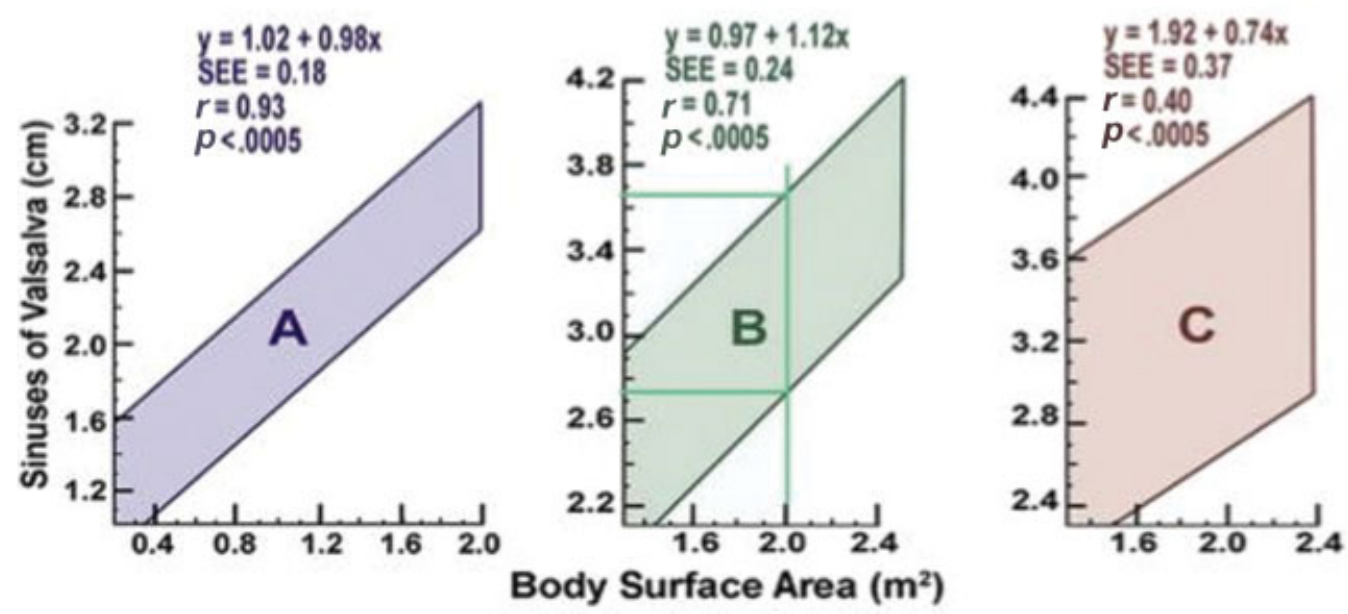

Fig. 3 Normal ranges of Sinuses of Valsalva diameter with respect to body surface area across three age groups (left to right: 1-15 years, 20-39 years, and $>40$ years). SEE, standard error of the estimate. Image courtesies: Roman et $\mathrm{l}^{5}$ and Goldstein et al. ${ }^{8}$

\begin{tabular}{|c|c|c|c|c|c|c|c|c|c|c|c|}
\hline & & \multicolumn{10}{|c|}{ Aortic Size (cm) } \\
\hline & & 3.5 & 4.0 & 4.5 & 5.0 & 5.5 & 6.0 & 6.5 & 7.0 & 7.5 & 8.0 \\
\hline \multicolumn{12}{|c|}{$\begin{array}{c}\text { Height } \\
\text { (inches) (m) }\end{array}$} \\
\hline 55 & $1.4 \theta$ & 250 & 2.86 & 3.21 & 3.57 & 3.93 & 48 & 404 & 50 & 546 & -0 \\
\hline 57 & 1.45 & 2.41 & 2.76 & 3.10 & 3.45 & 3.79 & A.14 & $4+x$ & $4 \times 3$ & (1) & 39 \\
\hline 59 & 1.50 & 2.33 & 2.67 & 3.00 & 3.33 & 3.67 & 4.00 & $1+11$ & $4 n 7$ & $5 \times n$ & 43 \\
\hline 61 & 1.55 & 2.26 & 2.58 & 2.90 & 3.23 & 3.55 & 3.87 & 110 & 4st & $4 \mu$ & 510 \\
\hline 63 & $1.6 \theta$ & 2.19 & 250 & 2.81 & 3.13 & 3.44 & 3.75 & 4.06 & at & 100 & $\sin$ \\
\hline 65 & 1.65 & 2.12 & 2.42 & 2.73 & 3.03 & 3.33 & 3.64 & 3.94 & 438 & 458 & $4 \mathrm{ks}$ \\
\hline 67 & 1.70 & 2.06 & 2.35 & 2.65 & 2.94 & 3.24 & 3.53 & 3.82 & 412 & 44 & 47 \\
\hline 69 & 1.75 & 2.00 & 2.29 & 2.57 & 2.86 & 3.14 & 3.43 & 3.71 & 4.00 & $14 x$ & $4: 97$ \\
\hline 71 & $1.8 \theta$ & 1.94 & 2.22 & 2.50 & 2.78 & 3.06 & 3.33 & 3.61 & 3.89 & $4 n$ & 4.46 \\
\hline 73 & 1.85 & 1.89 & 2.16 & 2.43 & 2.70 & 2.97 & 3.24 & 3.51 & 3.78 & 4.05 & 142 \\
\hline 75 & 1.99 & 1.84 & 2.11 & 2.37 & 2.63 & 2.89 & 3.16 & 3.42 & 3.68 & 3.95 & $4 \geq 1$ \\
\hline 77 & 1.95 & 1.79 & 2.05 & 2.31 & 2.56 & 2.82 & 3.08 & 3.33 & 3.59 & 3.85 & 410 \\
\hline 79 & 2.00 & 1.75 & 2.00 & 2.25 & 250 & 2.75 & 3.00 & 3.25 & 3.50 & 3.75 & 4.00 \\
\hline 81 & 2.05 & 1.71 & 195 & 2.20 & 2.44 & 2.68 & 293 & 3.17 & 3,41 & 3.66 & 390 \\
\hline
\end{tabular}

Fig. 4 Risk of complications (aortic dissection, rupture, and death) in patients with ascending aortic aneurysm as a function of aortic diameter (horizontal axis) and height (vertical axis), with the aortic height index given within the figure. Image courtesy: Zafar et al. ${ }^{7}$

aorta measurements should be done from leading edge to leading edge. For TTE, this means measurement of the outside of the anterior wall to the inside of the posterior wall, and for TEE, this would be reversed since the probe is behind the heart, that is, outside of the posterior wall to the inside of the anterior wall ( $\mathbf{- F i g} \mathbf{F A}, \mathbf{B}$ ). Leading edge to leading edge was originally recommended by the American Society of Echocardiography (ASE) in 1978 due to the belief that this technique reduces inaccuracies in measurement that could arise from blooming artifact due to the aortic wall being a bright reflector. ${ }^{9}$ Large studies that were done after those ASE recommendations and multiple guidelines have since used the leading edge to leading edge in end-diastole technique, and thus most available prognostic data have stemmed from this convention. Moreover, Muraru et $\mathrm{al}^{10}$ performed aortic measurements using inner edge to inner
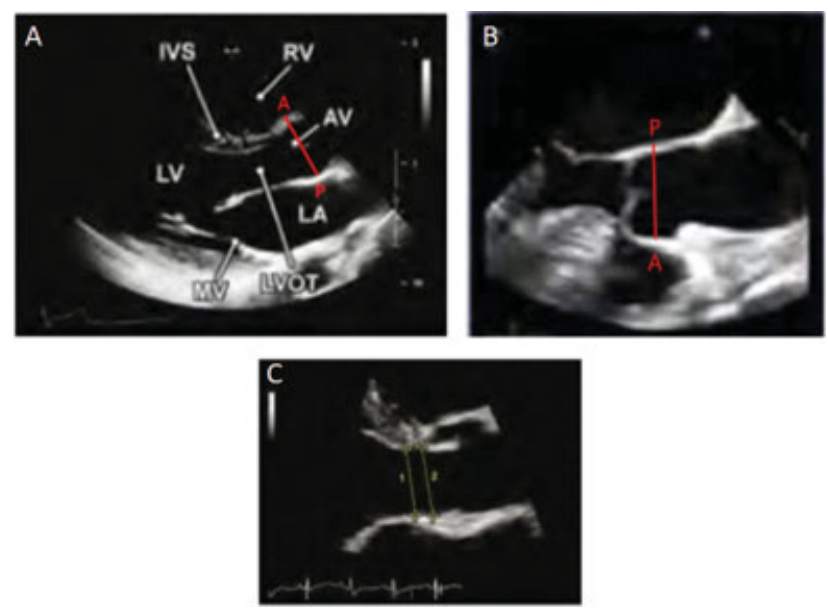

Fig. 5 (A) Parasternal long axis on transthoracic echo (TTE) showing leading edge to leading edge measurement, from outside the anterior wall to the inside of the posterior wall, (B) transesophageal echo (TEE) midesophageal view at 135 degrees (long axis) showing leading edge to leading edge measurement, from outside the posterior wall to the inside of the anterior wall, (C) parasternal long axis on TTE showing inner edge to inner edge measurement of the (1) LVOT and (2) aortic annulus in midsystole with open leaflets. A, anterior, AV, aortic valve; IVS, interventricular septum; LA, left atrium; LV, left ventricle; LVOT, left ventricular outflow tract; MV, mitral valve; P, posterior; RV, right ventricle. Red lines added for this figure to demonstrate proper measuring technique. Image courtesy: Mitchell et al. ${ }^{3}$

edge verses leading edge to leading edge with two-dimensional (2D) echocardiography in 218 healthy volunteers and found that inner edge measurements produced significantly smaller diameters as compared with leading edge diameters. ${ }^{10}$ The only exception to this convention, however, is the aortic annulus, which is measured from inner edge to inner edge, where the leaflets insert, in midsystole when the leaflet tips are open (-Fig. 5C). Finally, the TTE window that measurements are done is the PLAX, where the aorta lies perpendicular to the ultrasound probe.

Echocardiography is a useful imaging modality because it is cost effective, portable, and widely available. Other than echocardiography, however, computed tomography (CT) and magnetic resonance imaging (MRI) can also be used as 
multimodal approaches to imaging the aorta, though these techniques may not be as cost effective or accessible. CT angiography (CTA) can provide a rapid, noninvasive tool that allows visualization of the entire aorta, though it exposes the patient to radiation and contrast and cannot provide functional information about the aortic valve. MRI can also provide visualization of the entire aorta without any radiation exposure, though it is costly and less widely available, and the patient has to remain still in a supine position for a much longer time. Interestingly, these imaging modalities use an inner edge to inner edge measuring technique. When comparing echocardiographic measurement convention, there is good correlation with inner edge to inner edge measurements in CT/MRI. In fact, the three modalities were compared by Rodríguez-Palomares et $\mathrm{al}^{11}$ in 2016 in a study of 140 patients with aortic disease. They found that when comparing leading edge to leading edge technique in TTE to inner edge to inner edge technique in CT/MRI, there was good accuracy and reproducibility of aortic diameter estimation. When inner edge to inner edge was used in TTE, however, it underestimated the diameters as compared with $\mathrm{CT} / \mathrm{MRI}$. It is therefore worth highlighting that in addition to TTE, CT and MRI can be useful tools for physicians to utilize in aortic evaluation, especially when visualization of the entire aorta is necessary as this cannot be achieved with TTE alone.

\section{Echocardiographic Evaluation of Selected Aortic Pathologies}

\section{Aortic Dissection}

Aortic dissection, which falls under the category of acute aortic syndromes, occurs when there is a tear in the intimal layer of the aorta, and pulsatile blood flow enters causing a separation of the layers. Subsequently, the tear can propagate, resulting in two lumens of blood flow, a true and false lumen. Aortic dissections can be categorized based on the Stanford classification system into Type A and Type B, with Type A defined as dissections of the ascending aorta \pm the arch and descending aorta, and Type B defined as dissection of the aorta below the level of the left subclavian artery only. ${ }^{12}$ Type A dissections are particularly dangerous, as they can propagate into the coronary arteries causing myocardial infarction, disrupt the aortic valve resulting in significant valvular regurgitation, or extend into the pericardium resulting in acute pericardial effusion and cardiac tamponade. While CT has traditionally been the initial diagnostic test of choice, echocardiography provides valuable information in this condition, and the two modalities can be used in combination for evaluation and treatment planning. CT has the advantage that it can allow visualization of the entire aorta and branch vessels, but it does not provide any functional data that echocardiography can provide such as the mechanism and severity of aortic regurgitation, presence of wall motion abnormalities to suggest coronary involvement, or evidence of hemodynamically significant pericardial effusion. Furthermore, in patients who are hemodynamically too unstable to go to a CT scanner, or cannot get contrast due to renal disease, TEE provides a portable diagnostic tool that can be performed at the bedside with a sensitivity of 86 to $100 \%$ and a specificity of 90 to $100 \%{ }^{13}$ It is important to keep in mind, however, that TEE has a blind spot at the distal ascending aorta and proximal aortic arch because the airfilled tracheal carina interferes with ultrasound beam propagation from the esophagus to the heart. - Table 1 describes

Table 1 Role of echocardiography in detecting evidence of aortic dissection and echocardiographic definitions of main findings (adapted from Goldstein et $\mathrm{al}^{8}$ )

\begin{tabular}{|c|c|}
\hline Diagnostic goals & Definition by echocardiography \\
\hline Identify presence of a dissection flap & Flap diving two lumens \\
\hline Define extension of aortic dissection & $\begin{array}{l}\text { Extension of the flap and true/false lumens in the aortic root (ascending/arch/ } \\
\text { descending abdominal aorta) }\end{array}$ \\
\hline Identify true lumen & $\begin{array}{l}\text { Systolic expansion, diastolic collapse, systolic jet directed away from the lumen, } \\
\text { absence of spontaneous contrast, and forward systolic flow }\end{array}$ \\
\hline Identify false lumen & $\begin{array}{l}\text { Diastolic diameter increase, spontaneous contrast and or thrombus formation, and } \\
\text { reverse/delayed or absent flow }\end{array}$ \\
\hline $\begin{array}{l}\text { Identify presence of false } \\
\text { luminal thrombosis }\end{array}$ & Mass separated from the intimal flap and aortic wall inside the false lumen \\
\hline Localize entry tear & $\begin{array}{l}\text { Disruption of the flap continuity with fluttering or ruptured intimal borders; color } \\
\text { Doppler shows flow through the tear }\end{array}$ \\
\hline $\begin{array}{l}\text { Assess presence, severity and } \\
\text { mechanisms of aortic regurgitation }\end{array}$ & $\begin{array}{l}\text { Anatomic definition of the valve (bicuspid, degenerated, and normal with/without } \\
\text { prolapse of one cusp); dilation of different segments of the aorta; flap invagination } \\
\text { into the valve; and severity by classic echocardiographic criteria }\end{array}$ \\
\hline Assess coronary artery involvement & $\begin{array}{l}\text { Flap invaginated into the coronary ostium, flap obstructing the ostium, absence of } \\
\text { coronary flow, and new regional wall motion abnormalities }\end{array}$ \\
\hline Assess side-branch involvement & Flap invaginated into the aortic branches \\
\hline Detect pericardial and/or pleural effusion & Echo-free space in the pericardium/pleura \\
\hline $\begin{array}{l}\text { Detect signs of } \\
\text { cardiac tamponade }\end{array}$ & Classic echocardiographic and Doppler signs of tamponade \\
\hline
\end{tabular}




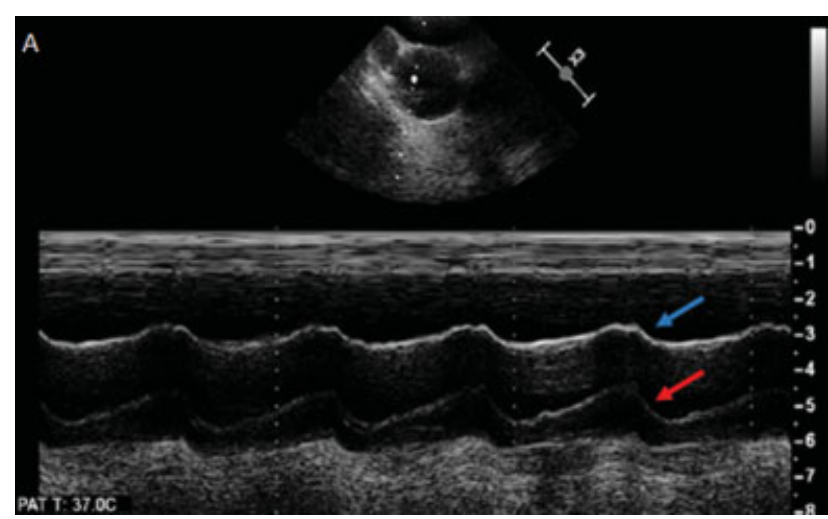

Fig. $6 \mathrm{M}$-mode of the aorta in cross section on transesophageal echo showing reverberation artifact. The "dissection flap" (red arrow) shows perfect concordance with the aortic wall (blue arrow), suggesting artifact rather than true dissection. Image courtesy: Yale Echocardiography Laboratory.

the different criteria for diagnosis of dissection that can be detected by echocardiography.

One of the pitfalls of aortic imaging by echocardiography to note is reverberation artifact which can create the false appearance of a dissection flap. A reverberation artifact occurs when there is a strong reflector of ultrasound beams within the scanning sector, such as the wall of the aorta, which causes the ultrasound beams to bounce back and forth causing the initial structure to reappear with weaker intensity, farther away from the probe. ${ }^{14}$ This can result in a diagnostic dilemma in which the reader may confuse an artifact for a dissection flap. There are several tips and tricks that can be used to distinguish artifact from true dissection. Color Doppler imaging can help with this distinction, as it will respect the boundaries of a true dissection flap and show two distinct areas of different color flow in the true lumen and the false lumen (-Video 1A, B; available in the online version only). ${ }^{15}$ If the false lumen is thrombosed, it may show minimal or no color flow. However, color Doppler flow patterns will traverse the entirety of the aortic lumen without respecting a "flap" that is really reverberation artifact ( - Video 1C, D; available in the online version only). Mmode interrogation can also be performed through the aorta to see if there is independent motion of the "flap." A reverberation artifact will not demonstrate independent motion and will perfectly mimic movement of the aortic wall, whereas a true dissection flap should demonstrate independent fluttering motion as blood flows through it (-Fig. 6).

\section{Video 1}

(A) Transesophageal echo (TEE) view of the aorta in transverse cross section showing a dissection flap with a true lumen and a false lumen that is thrombosed, (B) TEE view from - Video 1 A with color Doppler, showing respect of the flap boundaries and no flow in the thrombosed false lumen, (C) TEE view of the aorta in transverse cross section showing a reverberation artifact "flap," (D) TEE view from - Video 1C with color Doppler, showing color traversing the entirety of the lumen. Videos from the Yale Echocardiography Laboratory. 2D, two-dimensional; bpm, beats per minute. Online content including video sequences viewable at: https://www. thieme-connect.com/products/ejournals/html/10.1055/ s-0041-1724005.

Lastly, contrast agents can be used to follow blood flow, and visualization of different lumens can be identified. Similar to color flow, contrast will either follow or not follow the boundaries of the flaps in the cases of true dissection versus artifact, respectively. While TTE has less sensitivity than TEE for dissection, the addition of contrast can increase TTE sensitivity from $70-85$ to $93 \%$ assuming sufficient image quality. ${ }^{8,13}$

\section{Bicuspid Aortic Valve}

Bicuspid aortic valve (BAV) is a congenital cardiac abnormality in which there are two aortic valve cusps instead of three. It has a prevalence of 1 to $2 \%$, with the most common type being fusion of the right and left coronary cusp, accounting for 70 to $80 \%$ of BAVs. ${ }^{16}$ Diagnosis of this condition is of paramount importance because these valves can degenerate at a much younger age leading to symptomatic stenosis or regurgitation, and patients can have associated aortic aneurysms with literature reports of prevalence varying from 20 to $84 \%{ }^{16}$ The primary mode of diagnosis of BAV is echocardiography. The best view to diagnose BAV is in the parasternal short axis. It is
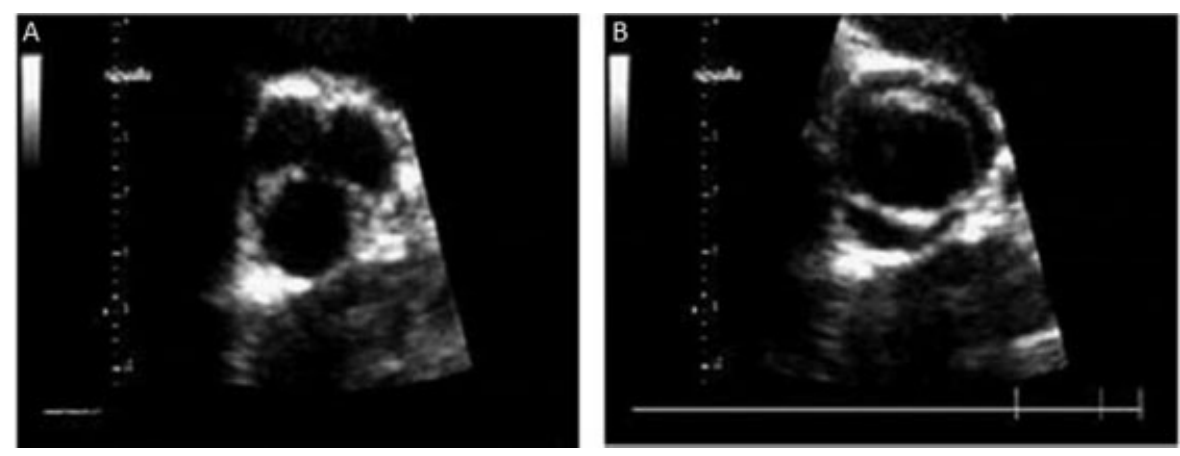

Fig. 7 (A) Aortic valve during diastole showing the appearance of three leaflets, (B) same aortic valve in systole showing true bicuspid valve. Image courtesy: Santarpia et al. ${ }^{17}$ 

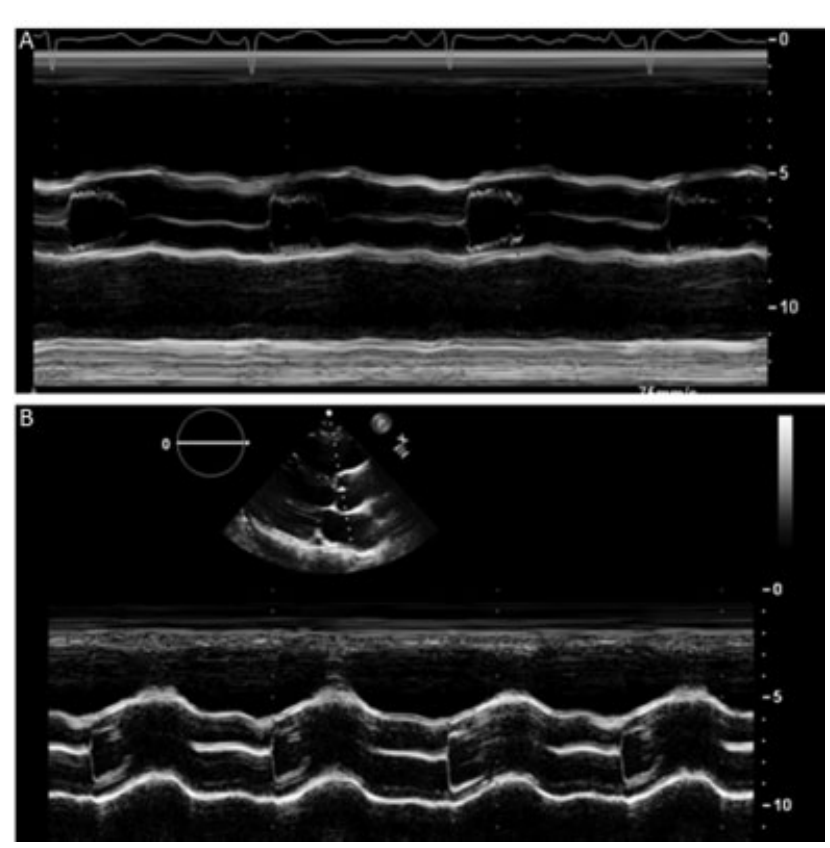

Fig. 8 (A) Central closure suggestive of a tricuspid aortic valve, (B) eccentric closure suggesting bicuspid aortic valve. Image courtesy: Yale Echocardiography Laboratory.

important to highlight that one pitfall is viewing the valve in diastole as opposed to systole. If it is visualized in diastole when the valve is closed, it can still appear trileaflet even if it is bicuspid due to the presence of a raphé or fusion line between two leaflets. Therefore, it should be viewed in systole when the valve is open, to visualize the commissural fusion with raphé and "fish mouth" or football-shaped appearance of the valve orifice (-Fig. 7). ${ }^{17}$

Another tip to help distinguish bicuspid from trileaflet valve is M-mode interrogation through the aortic valve in PLAX. Normally, the closure line of the aortic valve in diastole should be in the center of the aorta, but with bicuspid valves, one of two valve leaflets is usually bigger, and so the closure line will be eccentric (-Fig. 8A, B) ${ }^{18}$ Furthermore, in the PLAX, doming of the leaflets can be seen (-Video 2A; available in the online version only). Finally, a tip off suggesting possible bicuspid valve is eccentric aortic regurgitation ( - Video 2B; available in the online version only).

\section{Video 2}

(A) Parasternal long axis transthoracic echo (TTE) view showing doming of the aortic valve leaflets, (B) parasternal long axis TTE view with color Doppler showing an eccentric jet of aortic regurgitation. Source Yale Echocardiography Laboratory. Online content including video sequences viewable at: https://www. thieme-connect.com/products/ejournals/html/ $10.1055 / \mathrm{s}-0041-1724005$.

\section{Conclusion}

Echocardiography is a vital imaging modality in the evaluation of the thoracic aorta. It is important to keep in mind standard techniques for visualization and measurements, such as leading edge to leading edge, end diastole, and appropriate window, to accurately diagnose and follow-up patients. Different techniques, such as M-mode, color Doppler flow, or contrast echocardiography, can be useful tools to help distinguish between true disease and artifact. Clinicians should be aware of pitfalls in interpreting echocardiographic studies that may lead to misdiagnosis or delay in diagnosis. CT and MRI are other multimodal approaches for diagnosis and evaluation of aortic pathology when visualization on TTE or TEE is suboptimal or further imaging is required.

\section{Funding}

None.

\section{Conflict of Interest}

The authors declare no conflict of interest related to this article.

Acknowledgments

None.

\section{References}

1 Kuzmik GA, Sang AX, Elefteriades JA. Natural history of thoracic aortic aneurysms. J Vasc Surg 2012;56(02):565-571

2 LeMaire SA, Russell L. Epidemiology of thoracic aortic dissection. Nat Rev Cardiol 2011;8(02):103-113

3 Mitchell C, Rahko PS, Blauwet LA, et al. Guidelines for performing a comprehensive transthoracic echocardiographic examination in adults: recommendations from the american society of echocardiography. J Am Soc Echocardiogr 2019;32(01):1-64

4 Hahn RT, Abraham T, Adams MS, et al. Guidelines for performing a comprehensive transesophageal echocardiographic examination: recommendations from the American Society of Echocardiography and the Society of Cardiovascular Anesthesiologists. J Am Soc Echocardiogr 2013;26(09):921-964

5 Roman MJ, Devereux RB, Niles NW, et al. Aortic root dilatation as a cause of isolated, severe aortic regurgitation. Prevalence, clinical and echocardiographic patterns, and relation to left ventricular hypertrophy and function. Ann Intern Med 1987;106(06):800-807

6 Davies RR, Gallo A, Coady MA, et al. Novel measurement of relative aortic size predicts rupture of thoracic aortic aneurysms. Ann Thorac Surg 2006;81(01):169-177

7 Zafar MA, Li Y, Rizzo JA, et al. Height alone, rather than body surface area, suffices for risk estimation in ascending aortic aneurysm. J Thorac Cardiovasc Surg 2018;155(05):1938-1950

8 Goldstein SA, Evangelista A, Abbara S, et al. Multimodality imaging of diseases of the thoracic aorta in adults: from the American society of echocardiography and the European association of cardiovascular imaging: Endorsed by the society of cardiovascular computed tomography and society for cardiovascular magnetic resonance. J Am Soc Echocardiogr 2015;28(02):119-182

9 Sahn DJ, DeMaria A, Kisslo J, Weyman A. Recommendations regarding quantitation in M-mode echocardiography: results of a survey of echocardiographic measurements. Circulation 1978; 58(06):1072-1083

10 Muraru D, Maffessanti F, Kocabay G, et al. Ascending aorta diameters measured by echocardiography using both leading 
edge-to-leading edge and inner edge-to-inner edge conventions in healthy volunteers. Eur Heart J Cardiovasc Imaging 2014;15 (04):415-422

11 Rodríguez-Palomares JF, Teixidó-Tura G, Galuppo V, et al. Multimodality assessment of ascending aortic diameters: comparison of different measurement methods. J Am Soc Echocardiogr 2016; 29(09):819-826.e4

12 Gudbjartsson T, Ahlsson A, Geirsson A, et al. Acute type A aortic dissection - a review. Scand Cardiovasc J 2020;54(01):1-13

13 Evangelista A, Flachskampf FA, Erbel R, et al; European Association of Echocardiography Document Reviewers. Echocardiography in aortic diseases: EAE recommendations for clinical practice. Eur J Echocardiogr 2010;11(08):645-658

14 Bertrand PB, Levine RA, Isselbacher EM, Vandervoort PM. Fact or artifact in two-dimensional echocardiography: avoiding misdiagnosis and missed diagnosis. J Am Soc Echocardiogr 2016;29(05):381-391
15 Patil TA, Nierich A. Transesophageal echocardiography evaluation of the thoracic aorta. Ann Card Anaesth 2016;19(supplement): S44-S55

16 Borger MA, Fedak PWM, Stephens EH, et al. The American Association for Thoracic Surgery consensus guidelines on bicuspid aortic valve-related aortopathy: Full online-only version. J Thorac Cardiovasc Surg 2018;156(02):e41-e74

17 Santarpia G, Scognamiglio G, Di Salvo G, et al. Aortic and left ventricular remodeling in patients with bicuspid aortic valve without significant valvular dysfunction: a prospective study. Int J Cardiol 2012;158(03):347-352

18 Michelena HI, Prakash SK, Della Corte A, et al; BAVCon Investigators. Bicuspid aortic valve: identifying knowledge gaps and rising to the challenge from the International Bicuspid Aortic Valve Consortium (BAVCon). Circulation 2014;129(25): 2691-2704 